\title{
Digital Holography and 3D Imaging: introduction to the joint feature issue in Applied Optics and Journal of the Optical Society of America A
}

\author{
Pascal Picart, ${ }^{1, *}$ (i) Partha Banerjee, ${ }^{2}$ Marc Georges, ${ }^{3}$ Juan Liu, ${ }^{4}$ Liangcai Cao, ${ }^{5}$ \\ and Pierre-Alexandre Blanche ${ }^{6}$ \\ ${ }^{1}$ Le Mans Université, LAUM CNRS 6613, Avenue Olivier Messiaen, 72085 Le Mans, Cedex 9, France \\ ${ }^{2}$ Department of Electro-Optics and Photonics, University of Dayton, Dayton, Ohio 45469, USA \\ ${ }^{3}$ Centre Spatial de Liège, Université de Liège, Liege Science Park, B-4031 Angleur (Liège), Belgium \\ ${ }^{4}$ Beijing Institute of Technology, Beijing 100811, China \\ ${ }^{5}$ State Key Laboratory of Precision Measurement Technology and Instruments, Department of Precision Instrument, \\ Tsinghua University, Beijing 100084, China \\ ${ }^{6}$ College of Optical Sciences, The University of Arizona, Tucson, Arizona 85721, USA \\ *Corresponding author: pascal.picart@univ-lemans.fr
}

Received 19 November 2019; posted 19 November 2019 (Doc. ID 383909); published 26 November 2019

\begin{abstract}
The OSA Topical Meeting on Digital Holography and 3D Imaging (DH) was held 20-23 May 2019 in Bordeaux, France. Feature issues based on the DH meeting series have been released by Applied Optics (AO) since 2007. This year, $\mathrm{AO}$ and the Journal of the Optical Society of America A (JOSA A) jointly decided to have one such feature issue in each journal. This feature issue includes 46 papers in AO and 9 in JOSA A and covers a large range of topics, reflecting the rapidly expanding techniques and applications of digital holography and 3D imaging. The upcoming DH Conference (DH 2020) will be held from 22 to 26 June in Vancouver, Canada, as part of the OSA Imaging and Applied Optics Congress. ( 2019 Optical Society of America
\end{abstract}

https://doi.org/10.1364/JOSAA.36.000DH1

It has now become a tradition for every topical meeting on digital holography $(\mathrm{DH})$ to be succeeded by a feature issue in Applied Optics (AO). As in the case of DH 2017 and 2018, DH 2019 is being followed up with a joint issue between $\mathrm{AO}$ and the Journal of the Optical Society of America A (JOSA A). The topical meeting was held at the Institute of Optics Graduate School in Bordeaux, France, as a stand-alone conference. It had great success with 132 oral presentations (11 invited talks, two tutorials, two keynote addresses) and 79 posters, adding up to a grand total of 211.

The DH Topical Meeting is now recognized as the world's premier forum for science, technology, and applications of digital holography, 3D imaging, and display methods. In 2019, the meeting was organized around 22 sessions, including 2 poster sessions. Sessions addressed hot topics related to the areas of novel 3D imaging and display systems, computer-generated holograms, quantitative phase imaging, digital holographic microscopy, holographic tomography, holographic particle imaging, artificial intelligence and machine learning in digital holography, biomedical applications, and holography with various light sources, including gated, coherent, incoherent, and $\mathrm{X}$-ray to terahertz waves. Accordingly, this feature issue has a lot of excellent contributions, of which 46 are being published in $\mathrm{AO}$ and 9 in JOSA A. Six papers in AO and four in JOSA A are invited papers.

All submitted papers, including invited papers, have undergone peer review. We hope these articles will present state-of-the-art technological developments that are currently underway and stimulate further novel applications of digital holography and 3D imaging. The topical editors would like to thank all the contributors and the reviewers, without whom this feature issue would not have been possible. Finally, we wish the very best of success in the upcoming Digital Holography and 3D Imaging Conference, which will be held 22-26 June 2019 in Vancouver, Canada, as part of the OSA Imaging and Applied Optics Congress. 